\title{
Photoabsorption Spectra of Aqueous Oxyluciferin Anions Elucidated by Explicit Quantum Solvent
}

\author{
Yoshifumi Noguchi ${ }^{1}$, Miyabi Hiyama ${ }^{2}$, Motoyuki Shiga ${ }^{3}$, Osamu Sugino ${ }^{4}$, and \\ Hidefumi Akiyama ${ }^{4}$ \\ ${ }^{1}$ Department of Applied Chemistry and Biochemical Engineering, \\ Graduate School of Engineering, Shizuoka University, \\ Johoku 3-5-1, Hamamatsu, Shizuoka 432-8561, Japan \\ ${ }^{2}$ Graduate School of Science and Technology, Gunma University, 1-5-1 Tenjin-cho, \\ Kiryu, Gunma 376-8515, Japan \\ ${ }^{3}$ Center for Computational Science and E-Systems, Japan Atomic Energy Agency \\ 148-4 Kashiwanoha Campus, 178-4 Wakasiba, Kashiwa, Chiba, 277-0871, Japan \\ ${ }^{4}$ Institute for Solid State Physics, The University of Tokyo, \\ 5-1-5 Kashiwanoha, Kashiwa, Chiba 277-8581, Japan \\ E-mail: NOGUCHI.Yoshifumi@shizuoka.ac.jp
}

\section{CONTENTS}

Figure S1. Details of the Born-Oppenheimer first-principles simulations.

Figure S2. Simulations of the photoabsorption spectra.

Figure S3. QM/MM simulations.

Figure S4. Validity of the QM/MM.

Figure S5. BLYP Total energies simulated for keto, enol, and enolate in aqueous solution.

Figure S6. Radial distribution functions.

Figure S7. Radial distribution functions.

Figure S8. Photoabsorption spectra simulated by (a) full QM(B3LYP), (b) QM-MM with B3LYP, and (c) PCM with B3LYP.

Figure S9. Comparison of the photoabsorption spectra obtained from 100 and 1000 snapshots.

Figure S10. Fukui functions simulated for keto.

Figure S11. Fukui functions simulated for enol.

Figure S12. Fukui functions simulated for enolate. 


\section{S1. Details of the Born-Oppenheimer MD simulations}

We used the CPMD package and ran the Born-Oppenheimer first-principles MD simulations with a time step of $0.484 \mathrm{fs}$ for three oxyluciferin anions (phenolate-keto, phenolate-enol, and phenol-enolate) in a cubic box of $13.3723 \AA \times 13.3723 \AA \times 13.3723 \AA$. Then, we used the BLYP norm-conserving pseudopotential and the plane wave cut-off energy of $80 \mathrm{Ry}$ and added the van der Waals correction at the D2 level. The temperature was kept at $300 \mathrm{~K}$ by the Nose-Hoover thermostats. We only analyzed the converged data after 600 ps to discuss the stability of the oxyluciferin anion in the aqueous solution.

\section{S2. Simulations of the photoabsorption spectra}

We used the Gaussian09 package and simulated the photoabsorption spectra for 1000 randomly chosen structures. Of note, the same 1000 structures are employed for the full QM, QM/MM, and PCM (with a Gaussian09 default option) simulations. Then, we chose the nearest 64 water molecules with the oxyluciferin anion and transformed the coordinates from the periodic crystal coordinates, which were used in the MD simulations, to the molecular coordinates. Our photoabsorption spectra were simulated for an isolated cluster containing the oxyluciferin anion and 64 water molecules in the vacuum, which is different from the MD simulation under the periodic boundary condition.

\section{S3. QM/MM simulations}

For the QM/MM simulations with the CAM-B3LYP functional, we performed the natural population analysis with CAM-B3LYP+D3/6-311G* for 1000 structures to determine the point charges of $\mathrm{H}$ and $\mathrm{O}$ atoms of water molecules. By replacing all atoms of the water molecules with the point charges, we ran the QM/MM simulations for the same 1000 structures. Our QM/MM simulations treated the oxyluciferin anion as QM and 64 water molecules as MM. For the QM/MM with B3LYP, we repeated a similar procedure. However, B3LYP was used for the natural population analysis and for QM simulation. 


\section{S4. Validity of QM/MM}

In the "Validity of QM/MM" section, we performed QM/MM simulations with different sizes of QM systems including not only the oxyluciferin anion but also $n$ water molecules $(n=0,1,2,4,5,7,10$, and 30). We used the TIP3P water molecules instead of the NPA charge used in the previous section.

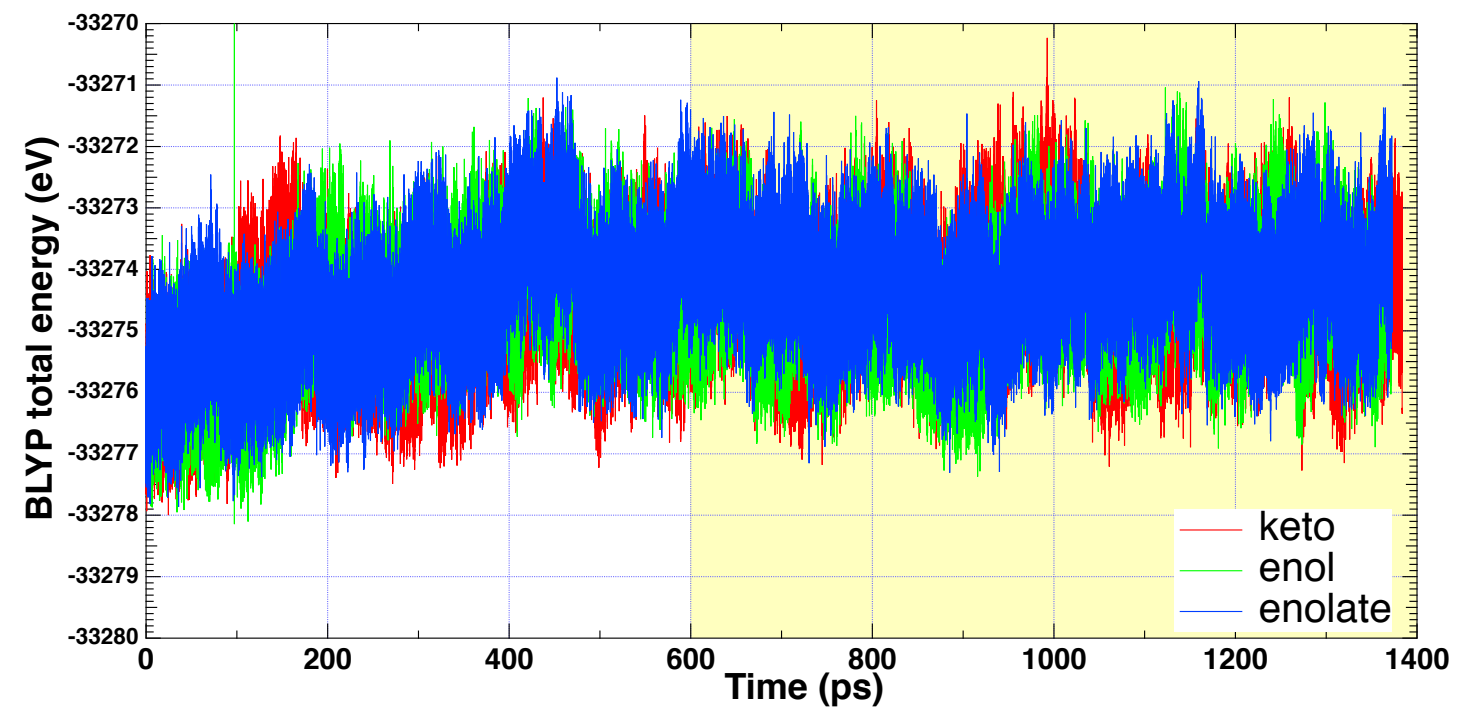

S 5. BLYP total energies. 

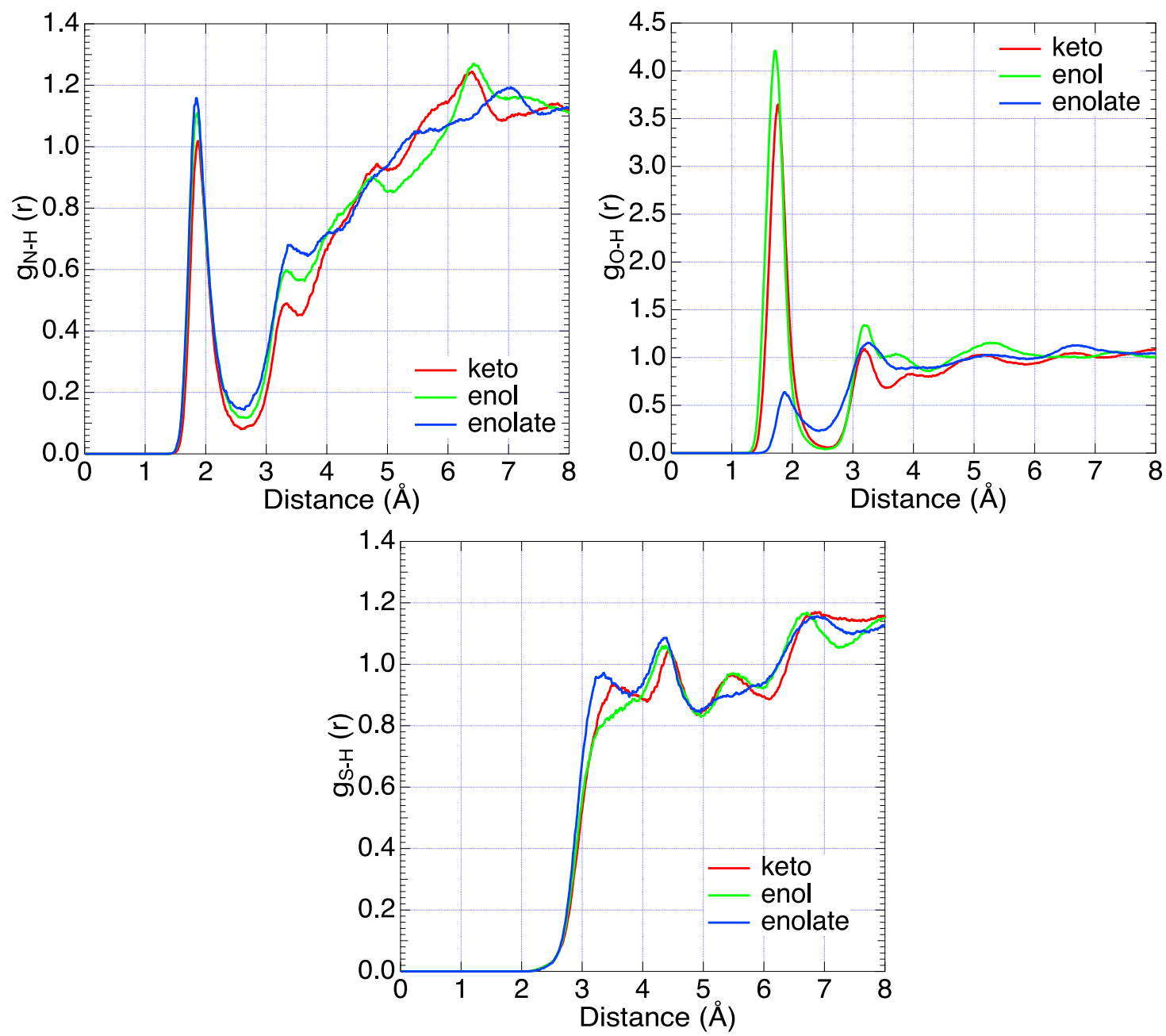

S 6. Radial distribution functions. 

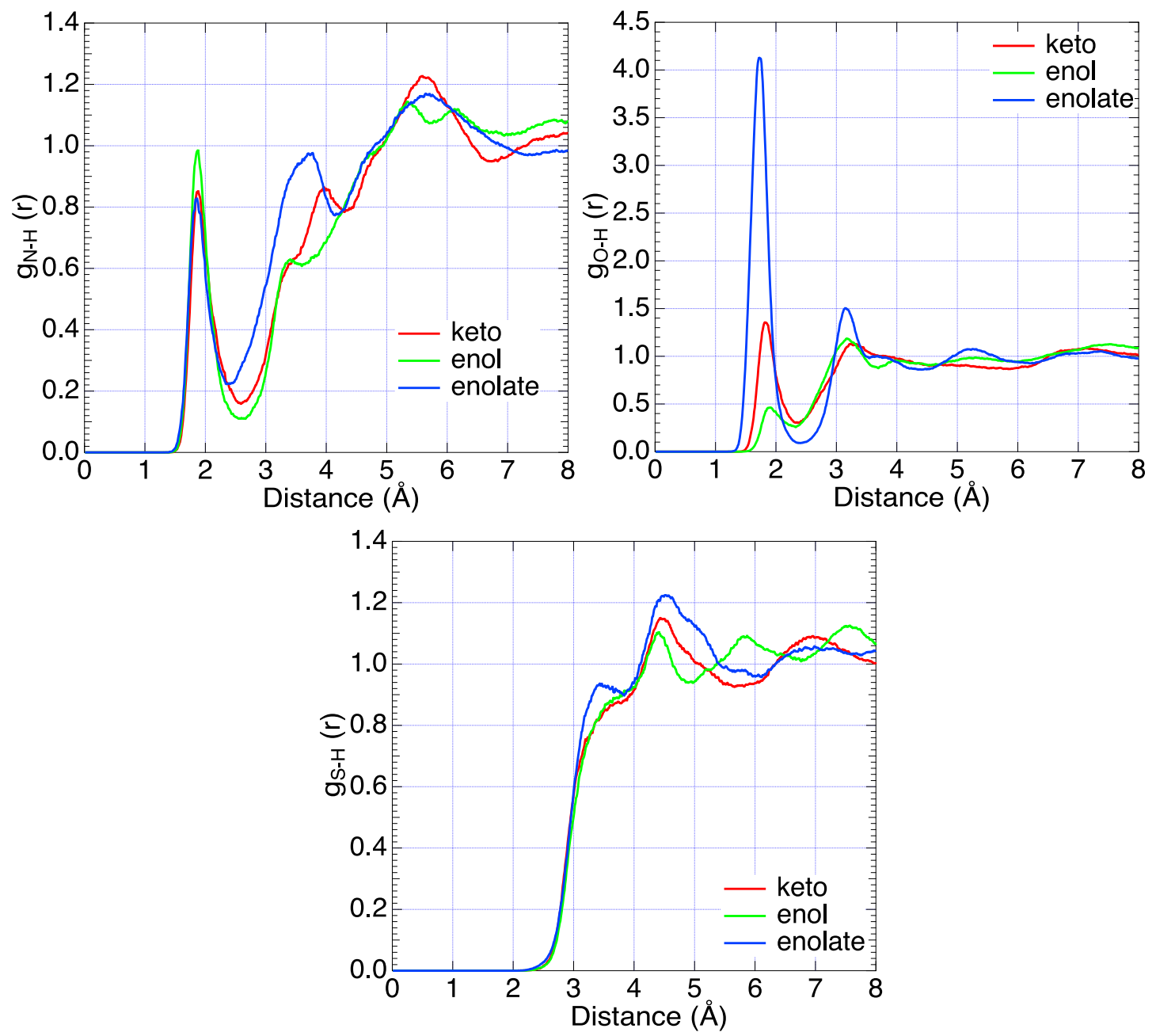

S 7. Radial distribution functions. 


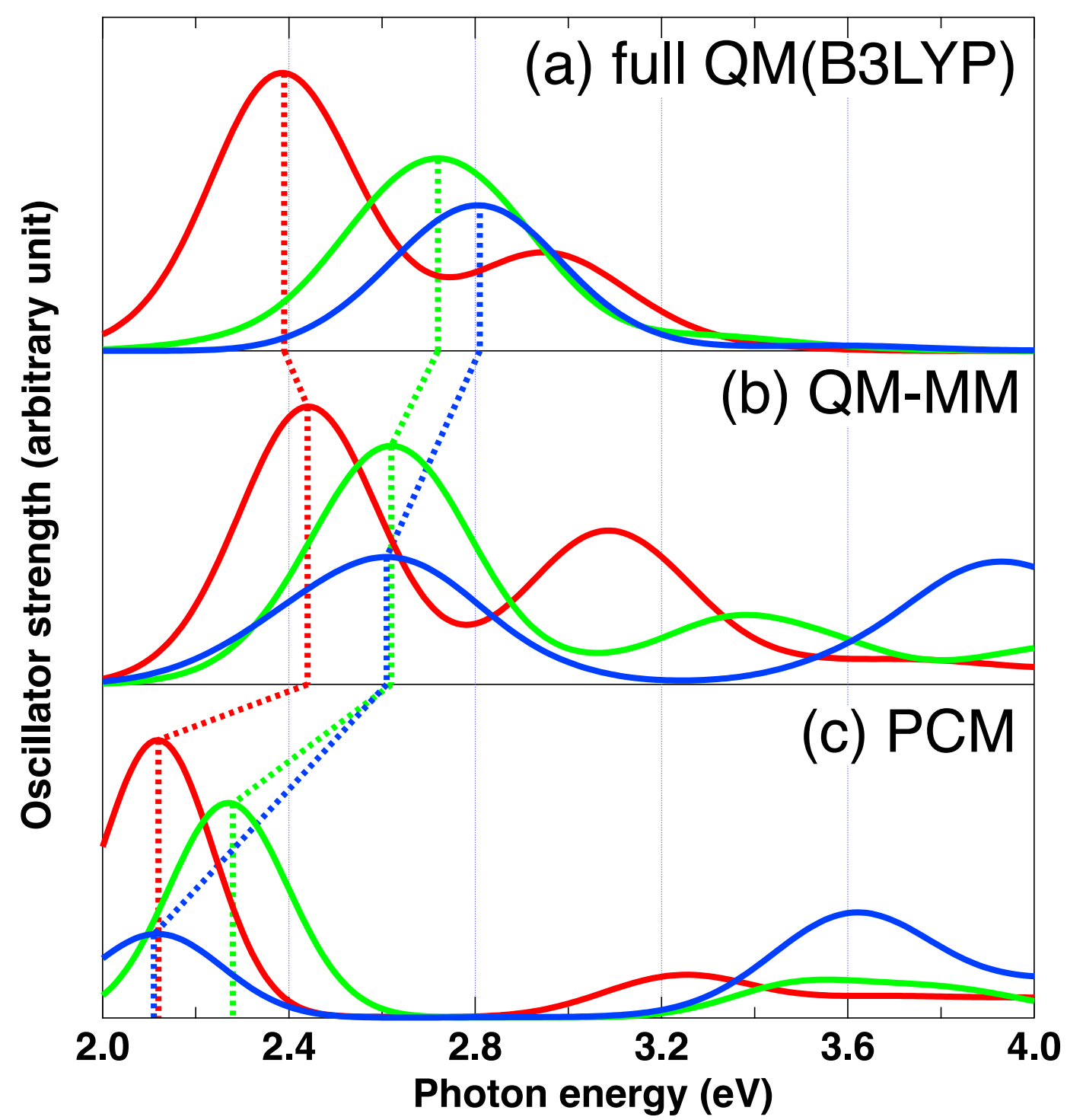

S 8. Photoabsorption spectra. 


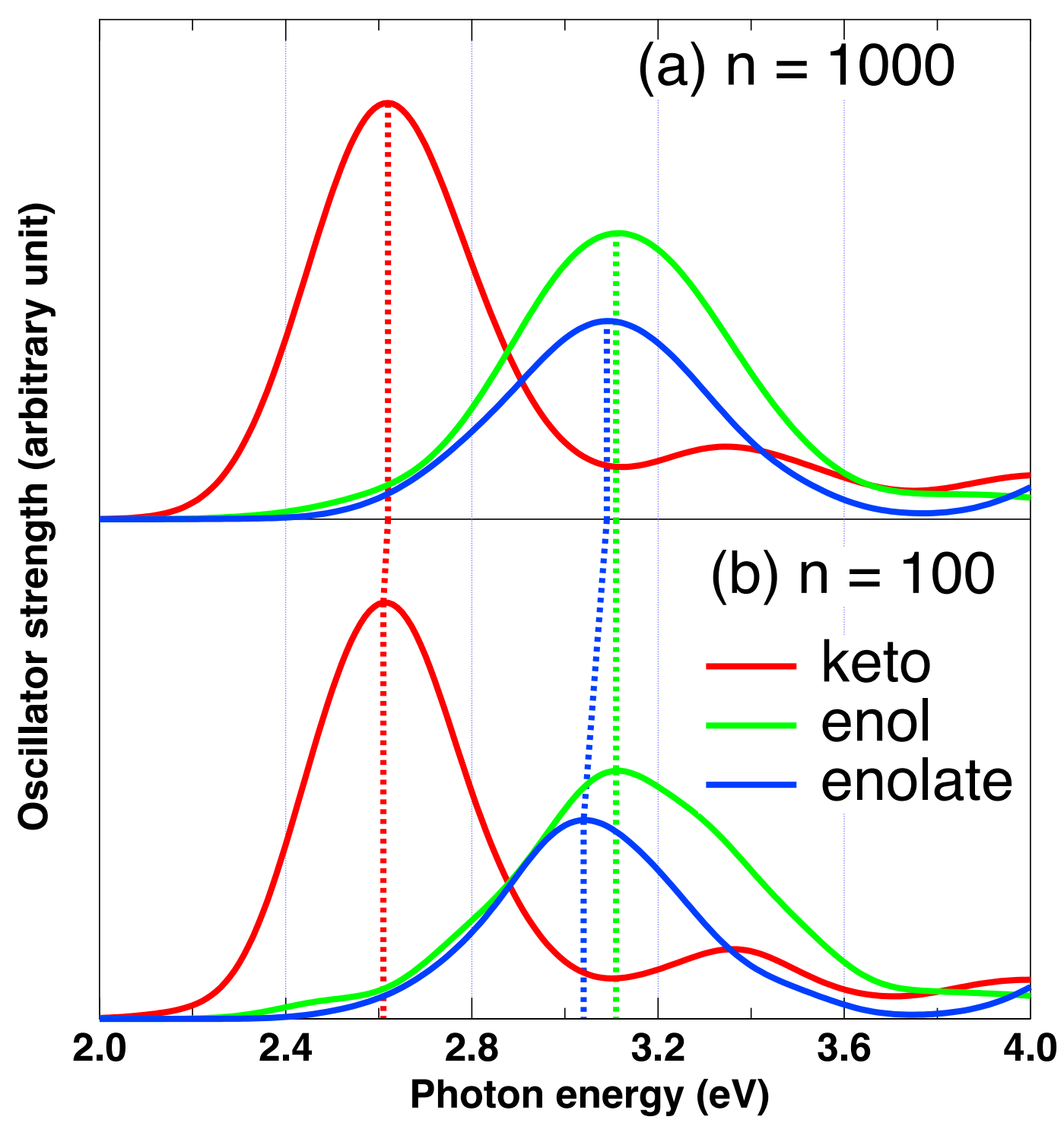

S 9. Photoabsorption spectra simulated with TD-CAM-B3LYP+D3/6-311G* for (a) 1000 and (b) 100 structures. 100 structures are not enough to converge the peak width and the peak heights. 

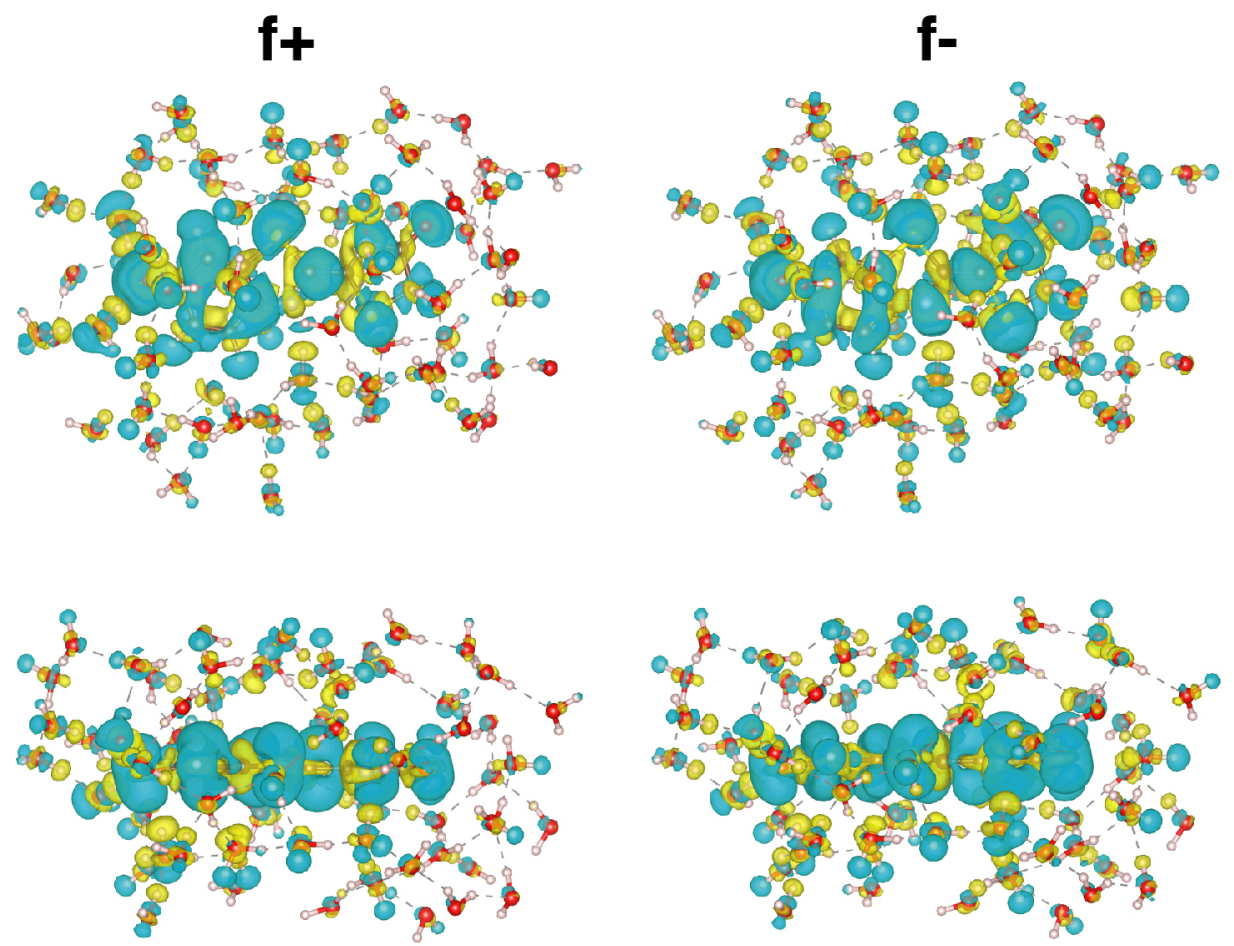

S 10. Fukui functions simulated for keto. $f_{+}(r)=\rho_{N}(r)-\rho_{N-1}(r), f_{-}(r)=\rho_{N-1}(r)-$ $\rho_{N-2}(r)$ 

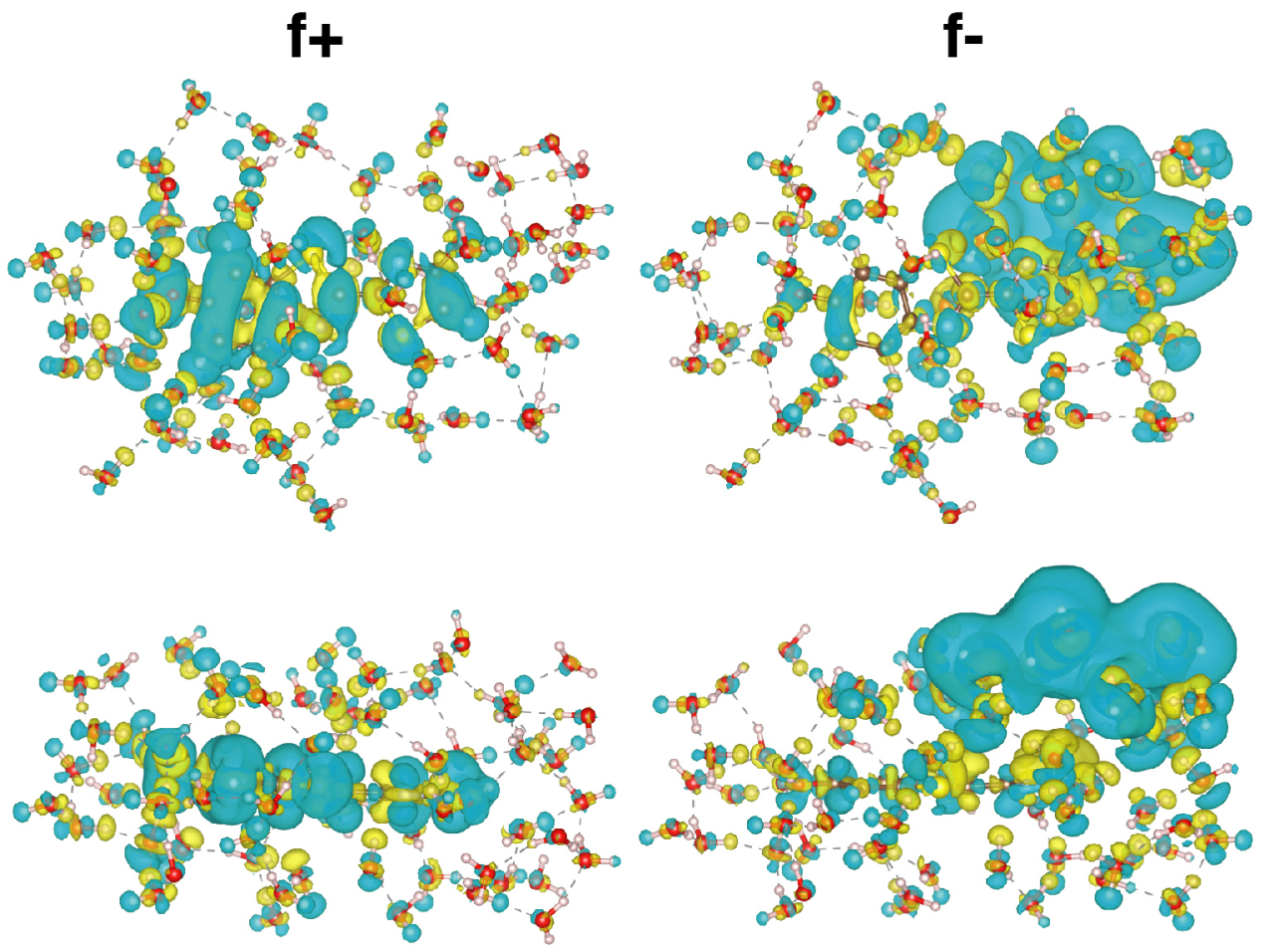

S 11. Fukui functions simulated for enol. $f_{+}(r)=\rho_{N}(r)-\rho_{N-1}(r), f_{-}(r)=\rho_{N-1}(r)-$ $\rho_{N-2}(r)$ 

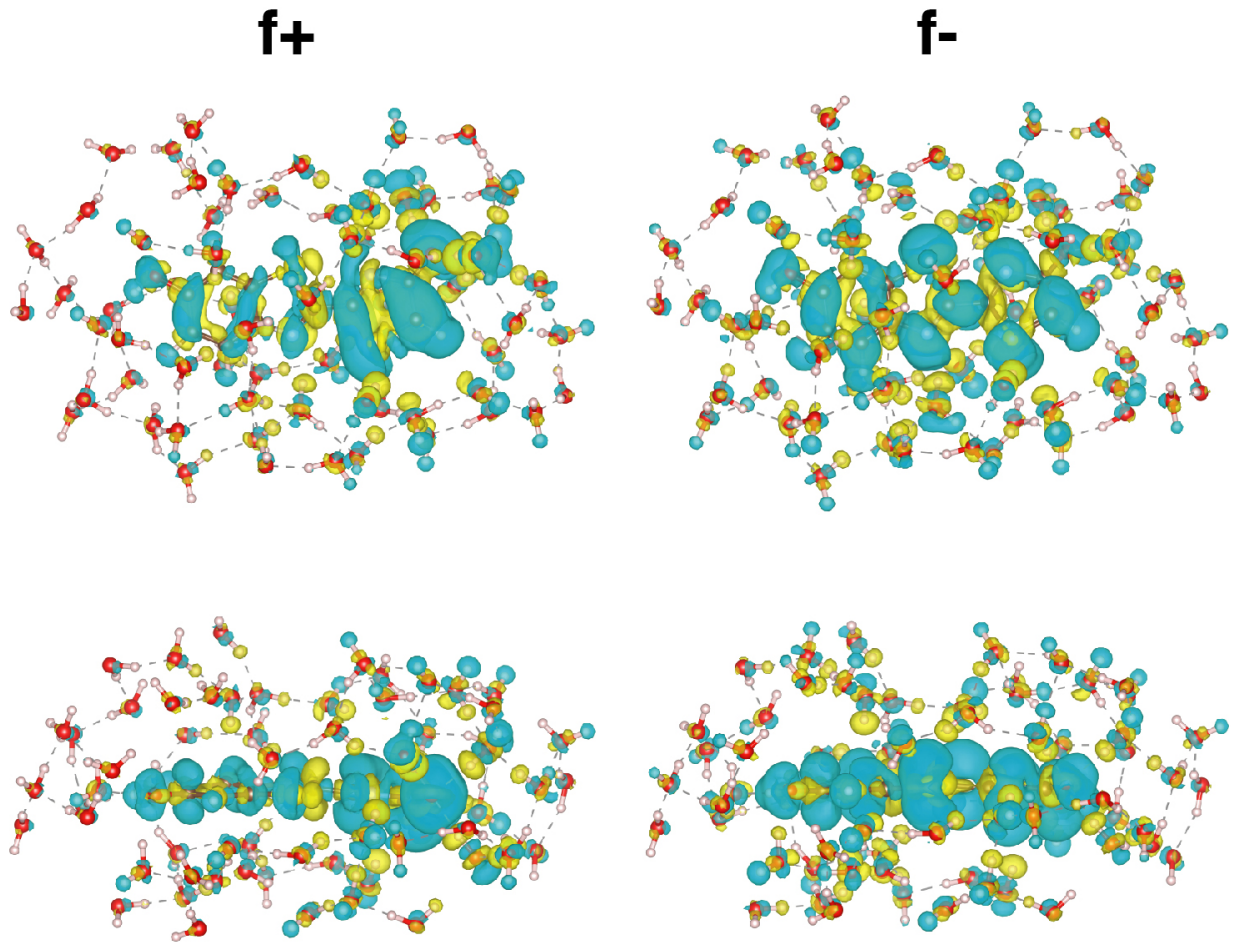

$\mathrm{S}$ 12. Fukui functions simulated for enolate. $f_{+}(r)=\rho_{N}(r)-\rho_{N-1}(r), f_{-}(r)=$ $\rho_{N-1}(r)-\rho_{N-2}(r)$ 